\title{
VIP-like Immunoreactive and Vasopressin-like Immunoreactive Neuronal Elements in the Suprachiasmatic Nucleus of Japanese Monkey (Macaca fuscata): Light and Electron Microscopic Immunocytochemical Study
}

\author{
Yasuhiko Ibata $^{1}$, Masaki Tanaka ${ }^{2}$, Norio Iijima ${ }^{2}$, Fumimasa Amaya ${ }^{3}$ and Yukio Ichitani ${ }^{4}$ \\ ${ }^{1}$ Office of the President, Department of ${ }^{2}$ Anatomy and ${ }^{3}$ Anesthesiology, Kyoto Prefectural University of Medicine, Kawaramachi- \\ Hirokoji, Kamikyo-ku, Kyoto 602-0841, Japan and ${ }^{4}$ Institute of Psychology and Behavioral Neuroscience, University of Tsukuba, \\ Tsukuba, Japan
}

Received May 23, 2003; accepted July 24, 2003

VIP-like immunoreactive (VIP-LIR) and vasopressin-like immunoreactive (VPLIR) neuronal elements located in the monkey suprachiasmatic nucleus (SCN) were investigated by light and electron microscopic immunocytochemistry. Both populations of neurons in the monkey SCN show a similar distribution to those of the rat except for some subtle differences. In addition to the ordinary distribution of VIP-LIR and VP-LIR neurons, VIP-LIR neurons of similar or some smaller size to those in the ventrolateral portion of the nucleus were also observed in the periventricular nucleus adjacent to the third ventricle. VP-LIR neurons with similar size and either strong or weak immunoreactivity compared with those located in the dorsomedial area were detected in the periventricular nucleus in which magnocellular VP-LIR neurons with strong immunoreactivity were distributed.

Both VIP-LIR and VP-LIR neuronal perikarya were found to contain well developed cell organelles such as rER and mitochondria as well as immunoreactive dense granules. VIP-LIR neuronal perikarya were generally surrounded by astroglial processes. However, nonimmunoreactive axons were frequently found to make synaptic contact with VIP-LIR dendrites or dendritic spines. Thus the VIP-LIR neurons are thought to receive neuronal inputs from other parts of CNS mainly from the dendrites or dendritic spines. VIP-LIR and VP-LIR axons were seen to form synapses on non-immunoreactive dendrites. VIP-LIR axons were also found to form axoaxonic synapses on non-immunoreactive axons. Synaptic contacts between VIP-LIR neuronal elements were also occasionally observed.

The present study has demonstrated that both the VIP-LIR and VP-LIR neurons are at least partly intrinsic neurons in the SCN, and also projecting elements to the other neurons of the CNS.

Key words: VIP, vasopressin, suprachiasmatic nucleus, fine structure, monkey

\section{Introduction}

The suprachiasmatic nucleus (SCN) of mammalian species is considered to be a circadian oscillator of several physiological phenomena such as sleep, wakefulness, locomotion and hormone release. Destruction of this nucleus induces a disorder of the circadian rhythmicity of the above

Correspondence to: Yasuhiko Ibata, M.D., Ph.D., Office of the President, Kyoto Prefectural University of Medicine, KawaramachiHirokoji, Kamikyo-ku, Kyoto 602-0841, Japan. mentioned physiological phenomena [10, 17, 23]. Neuronal perikarya, containing neuropeptides such as vasopressinlike immunoreactive (VP-LIR), vasoactive intestinal peptide-like immunoreactive (VIP-LIR) and somatostatin-like immunoreactive neurons, have been reported to exist in this nucleus in rodents [3, 20, 29]. Among these, VP-LIR and VIP-LIR neurons are the main neuronal components and they are located in specific regions of the SCN. VP-LIR neurons are localized in the dorsomedial portion whereas VIPLIR neurons are in the ventrolateral portion. It is also interesting that neuronal projections from other CNS areas such 
as the retina and the raphe nuclei are restricted to the ventral portion of the $\operatorname{SCN}[11,18,19]$. These inputs make synaptic contacts with the VIP-LIR neuronal elements [6, 9, 14, 26]. Moreover, it has been demonstrated that VIP-LIR neurons show a diurnal variation in their immunoreactivity [25] and messenger RNA (mRNA) [1, 5, 21], and that this variation appears to be under the influence of the neuronal inputs from the retina. Therefore we considered the VIP-LIR neurons to play a significant role in entrainment. On the other hand, mRNA of vasopressin has been shown to be altered via circadian rhythms [28]. To date, morphological and physiological experiments examining immunocytochemistry of the neuropeptides [3, 29] and in situ hybridization of mRNA in the SCN have been done using rodents.

In higher mammalian species, VIP-LIR and VP-LIR neurons in the human SCN have been elucidated [16, 24], but to our knowledge no investigation on those neurons in the primate SCN particulary their fine structures has ever been done. Therefore, in the present study, we examined the VIP-LIR and VP-LIR neuronal elements in the SCN of Japanese monkey (Macaca fuscata) using light and electron microscopic immunocytochemistry. A short report related to these two immunoreactive neuronal peptides, particularly the VIP-LIR neuronal elements, has been presented [7]

\section{Materials and Methods}

Seven young male and female Japanese monkeys (Macaca fuscata weighing about $4-5 \mathrm{~kg}$, around 5 years old from the inspection of tooth eruption) were used in this study. They were given feed for the monkey and housed under $12 \mathrm{hr}$ light and $12 \mathrm{hr}$ dark schedule. Three animals were used for light microscopic immunocytochemistry and other four animals for electron microscopic immunocytochemistry. All animals used in the present study were conducted according to the NIH guidelines for the care and use of laboratory animals. Experiments were also permitted by inspection of the committee of Animal Research, Kyoto Prefectural University of Medicine. They were injected with colchicine $(180 \mu \mathrm{g} / 30 \mu \mathrm{l})$ in a lateral cerebral ventricle under deep anesthesia with nembutal $(25 \mathrm{mg} / \mathrm{kg})$ and ketamine $(50 \mathrm{mg} / \mathrm{kg}), 48 \mathrm{hr}$ before sacrifice to promote the storage of VIP-LIR and VP-LIR substances in the neuronal perikarya. Following perfusion through the left cardiac ventricle with a fixative containing $4 \%$ paraformaldehyde, $0.2 \%$ picric acid and $0.35 \%$ glutaraldehyde buffered at 7.4 with $0.1 \mathrm{M}$ phosphate buffer (PB) under deep nembutal anesthesia $(50 \mathrm{mg} / \mathrm{kg})$ at about $14.00 \mathrm{hr}$, the brains were removed and further fixed with similar fixative. They were then placed in $0.1 \mathrm{M}$ phosphate buffer saline (PBS). The hypothalamus of each animal was dissected from the brain. Frontal frozen sections of the hypothalamus $(30 \mu \mathrm{m}$ in thickness) were made from the brains of the animals used for light microscopic immunocytochemistry and stored in PBS containing $0.1 \%$ Triton X-100 for $48 \mathrm{hr}$ at $4^{\circ} \mathrm{C}$. Serial sections of the same thickness were cut by microslicer in PBS from the brains of the animals and were used for electron microscopic immunocytochemistry.

For light microscopic immunocytochemistry, the sections were first incubated in 1\% bovine serum albumin for 1 $\mathrm{hr}$ at room temperature and then incubated in anti-VIP serum (dilution, 1:3000) and anti-VP serum (1:5000 Immuno Nuclear Inc) alternately for $72 \mathrm{hr}$ at $4^{\circ} \mathrm{C}$. They were next incubated in biotinylated anti-rabbit IgG solution (1:1000, Vector Laboratories Inc) overnight at room temperature and then incubated in avidin-biotin complex (ABC) solution (1:1000, Vector Laboratories Inc) for $2 \mathrm{hr}$ at room temperature. After immunoreaction, the sections were exposed for $10 \mathrm{~min}$ at room temperature to $50 \mathrm{mM}$ Tris- $\mathrm{HCl}$ buffer containing $0.02 \% 3.3^{\prime}$-diaminobenzidine $4 \mathrm{HCl}$ (DAB), $0.045 \%$ $\mathrm{H}_{2} \mathrm{O}_{2}$ and $0.3 \%$ nickel ammonium sulfate, mounted on glass slides and examined by light microscopy. As a control, the sections were reacted with primary antisera against VIP and VP that had been previously absorbed with synthetic VIP $(10-100 \mu \mathrm{g})$ and VP $(10-50 \mu \mathrm{g})$. No positive immunoreactivity was detected in the control sections.

For electron microscopic immunocytochemistry, the sections were incubated in 1\% bovine serum albumin for 1 $\mathrm{hr}$ at room temperature and then incubated in anti-VIP serum (1:3000) and anti-VP serum (1:8000) alternately for $96 \mathrm{hr}$ at $4^{\circ} \mathrm{C}$. They were next incubated in biotinylated anti-rabbit IgG solution (1:1000) for $2 \mathrm{hr}$ at room temperature and then incubated in $\mathrm{ABC}$ solution $(1: 1000)$ for $2 \mathrm{hr}$ at room temperature. Thereafter they were exposed to DAB containing $\mathrm{H}_{2} \mathrm{O}_{2}$ for $5 \mathrm{~min}$ at room temperature. After confirmation of the presence of VIP-LIR and VP-LIR neuronal elements by light microscopy, the sections were fixed in chilled $1 \% \mathrm{OsO}_{4}$ solution for $1 \mathrm{hr}$, dehydrated in graded acetone series and embedded in Epon 812 mixture. Semithin sections were examined by light microscopy, following which ultrathin sections were stained with uranyl acetate and evaluated in a JEM 200CX electron microscope.

\section{Results}

\section{Light microscopic immunocytochemistry}

Light microscopic immunocytochemistry of VIP-LIR and VP-LIR neurons has shown that the dimensions of monkey SCN are almost $800 \mu \mathrm{m}$ (mediolateral), $400 \mu \mathrm{m}$ (dorsoventral) and $700 \mu \mathrm{m}$ (rostrocaudal).

\section{VIP-LIR neuronal elements}

The most remarkable population of VIP-LIR neurons was found in the ventrolateral portion of the central SCN just above the optic chiasm (Fig. 1a, b). The neurons were oval in shape and about $18 \mu \mathrm{m} \times 10 \mu \mathrm{m}$ in size. Fewer neurons were observed in the rostral and caudal portions of the SCN compared with the central portion. A few bipolar VIP-LIR neurons, the size of which were similar to those seen in the SCN were also located along the third ventricle within the periventricular nucleus just dorsal to the SCN (Fig. 1c). VIPLIR neuronal soma were also found buried among nerve fibers in the optic chiasm. The neuronal processes of these 

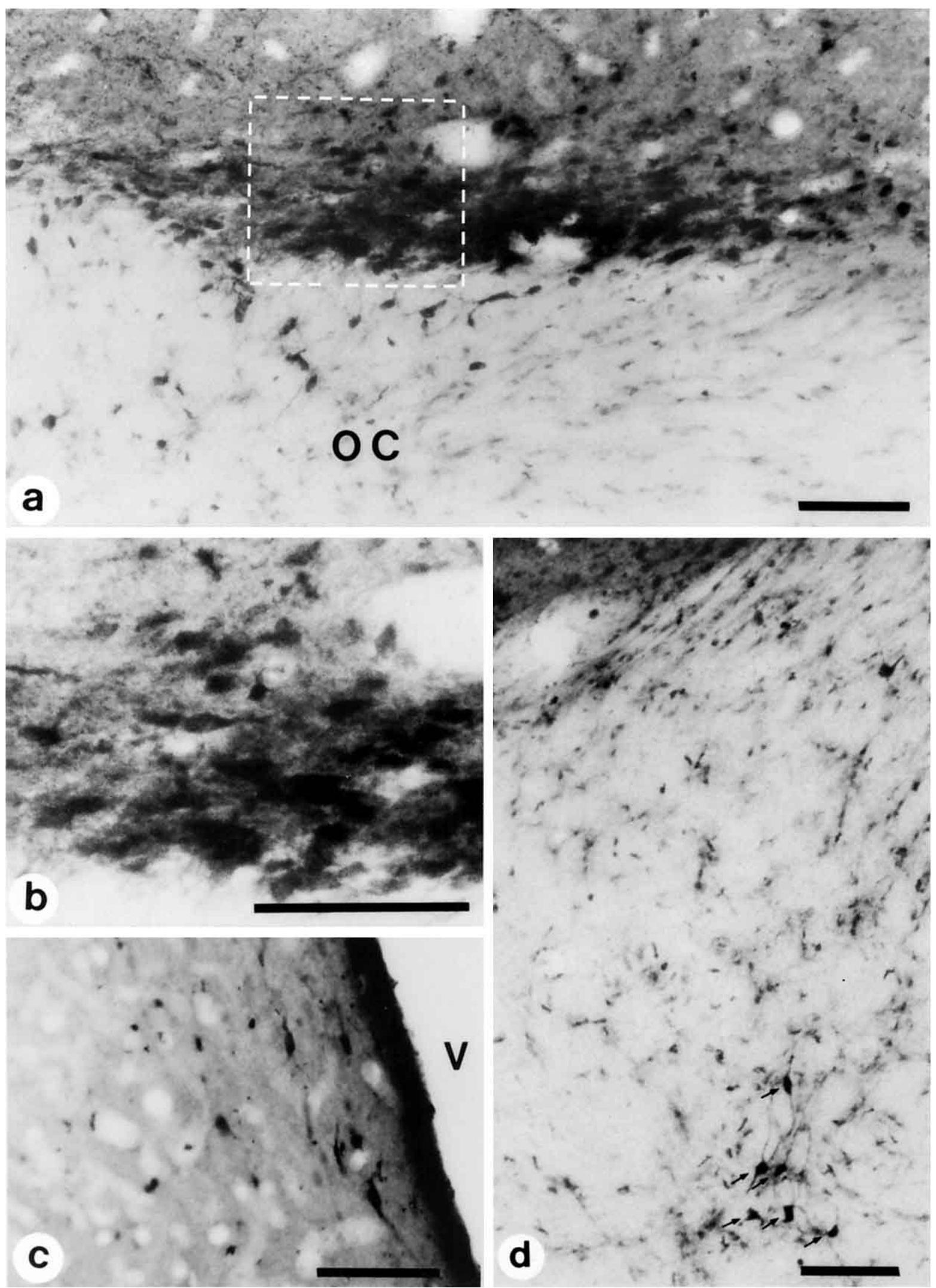

Fig. 1. a: VIP-LIR neuronal perikarya are densely packed in the ventrolateral portion of the central part of the SCN. Immunoreactive fibers are observed as dots. OC, optic chiasm. b: Higher magnification of VIP-LIR neurons in the square portion of a is shown. c: VIP-LIR neuronal perikarya with similar or smaller size to that of those in the ventrolateral portion of the SCN are detected in the periventricular nucleus adjacent to the dorsomedial portion of the SCN. V, third ventricle. d: VIP-LIR neuronal perikarya (arrow) are found among the optic nerve fibers in the optic chiasm at the level of rostral part of the SCN. Bar $=100 \mu \mathrm{m}$. 
cells could be traced dorsally to the basal portion of the SCN (Fig. 1d). VIP-LIR dots, presumed to be immunoreactive processes and terminals, were disseminated throughout the SCN particularly in the dorsal portion.

\section{VP-LIR neuronal elements}

Immunoreactivity of VP-LIR neurons in the SCN was far less than those seen in the paraventricular and supraoptic nuclei. In the central portion of the SCN, VP-LIR neurons were widely distributed coursing dorsomedially toward the periventricular nucleus (Fig. 2a). Moreover, a few of small VP-LIR neurons with either strong or weak immunoreactivity were also found intermingled with large and strongly immunoreactive VP-LIR neurons in the inferior portion of the periventricular nucleus (Fig. 2b). VP-LIR dots were also found around VP-LIR neuronal perikarya. In the rostral and caudal portion of the SCN, the distribution of VP-LIR neurons became sparse. In general, VP-LIR neurons were observed in the dorsomedial portion of the monkey SCN whereas VIP-LIR neurons were distributed in the ventral

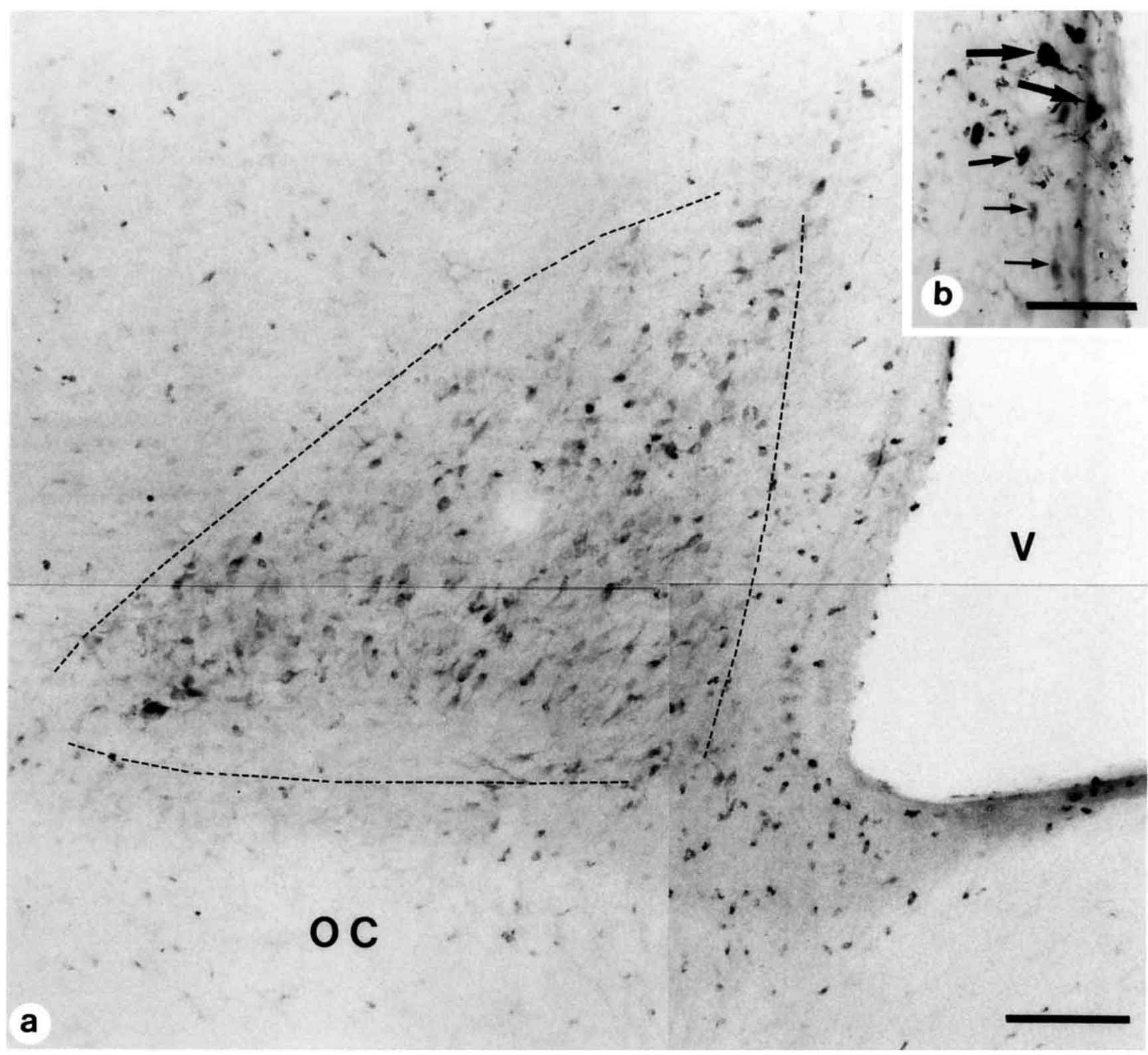

Fig. 2. a: The boundary of the SCN is shown by dotted triangle. VP-LIR neuronal perikarya are distributed in the central part of the SCN showing a trianglar distributional shape at the peak toward dorsomedial direction. However, they are not detected in the ventral portion which the VIP-LIR neuronal perikarya occupy. V, third ventricle; OC, optic chiasm. b: Large VP-LIR neuronal perikarya (large arrow) and small VP-LIR neuronal perikarya with strong (small thick arrow) or weak (small thin arrow) immunoreactivity are distributed in the periventricular nucleus. Large immunoreactive neurons are considered to be similar to magnocellular VP-LIR neurons in the paraventricular nucleus. Small strong or weak immunoreactive neurons are similar in size to that of those in the SCN. V, third ventricle. Bar=100 $\mu \mathrm{m}$. 


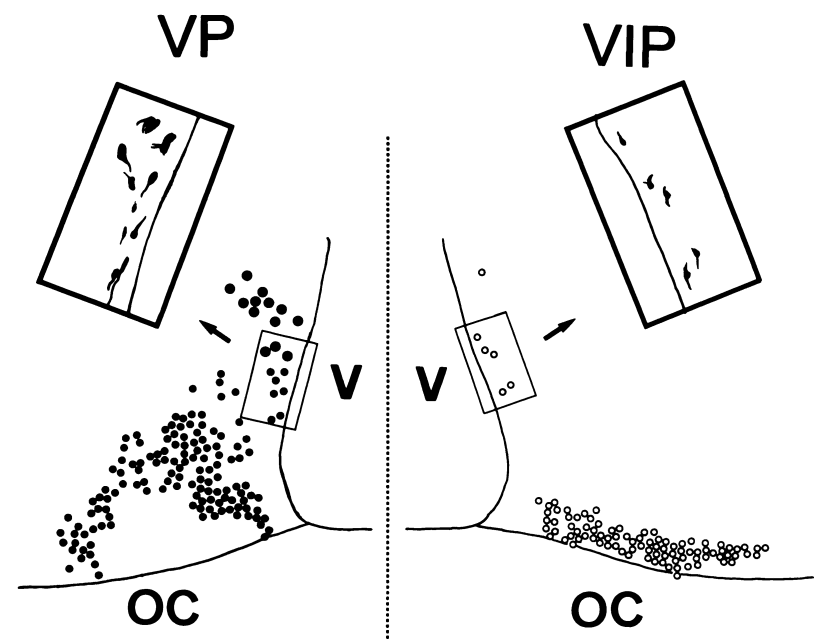

Fig. 3. Schematic drawing shows distributional pattern and density of VIP-LIR neurons (open circle) and VP-LIR neurons (closed circle) in the monkey SCN. The numbers of circles are equivalent to the population of both kinds of immunoreactive neurons. Open circles surrounded by a rectangle are VIP-LIR neurons distributed in the periventricular nucleus along the third ventricle. Their size is similar to that of VIP-LIR neurons in the SCN. Their features are shown by a camera lucida drawing (arrow). Large and small closed circles surrounded by a rectangle are magnocellular VP-LIR neurons with strong immunoreactivity and parvocellular VP-LIR neurons with strong or weak immunoreactivity in the periventricular nucleus. The size of parvocellular VIP-LIR neurons is similar to that of those in the SCN. Their features are described by a camera lucida drawing.

portion. This distributional pattern is schematically shown in Fig. 3.

\section{Electron microscopic immunocytochemistry \\ VIP-LIR neuronal elements}

VIP-LIR neurons have diffusely distributed chromatin in the nucleus, and fairly well developed cell organelles such as rER, polysomes and mitochondria. In addition to those organelles, VIP-LIR dense granules (100-120 nm in diameter) were disseminated throughout the cytoplasm (Fig. 4a). We confirmed that the majority of the VIP-LIR neuronal perikarya were surrounded by astroglial processes containing glial filaments (Fig. 4a). Thus, axo-somatic synapses were not readily detected on the surfaces of the VIP-LIR neuronal perikarya. Only a few axo-somatic synapses were seen terminating on a VIP-LIR perikaryon (Fig. 4b). VIPLIR neurons with well developed cell organelles were also seen surrounded by myelinated axons buried within the optic chiasm. VIP-LIR neuronal processes were also distributed here and there near the neuronal perikarya. VIP-LIR axons, dendrites and dendritic spines were broadly distributed throughout the SCN; however, their number seemed to be greater in the dorsal region. VIP-LIR axons containing synaptic vesicles, mitochondria and occasionally immunoreactive dense granules, the size of which was the same as found in the neuronal perikarya, were observed to make either asymmetrical or symmetrical synapses on non-immunoreactive dendrites (Fig. 5a). The number of asymmetrical synapses seemed to be larger than that of symmetrical synapses. VIP-LIR processes were occasionally found to make intimate contact with non-immunoreactive neuronal perikarya (Fig. 5b). Immunoreactive axons were also found to make asymmetrical axo-spinodendritic synapses on non-immunoreactive dendritic spines (Fig. 6a). Although the number was scarce, axo-axonic synapses between VIP-LIR and non-immunoreactive processes were detected (Fig. 6b). Occasionally VIP-LIR neuronal processes made synaptic contacts with each other (Fig. 6c). Non-immunoreactive axons of various sizes containing synaptic vesicles and mitochondria were observed to make symmetrical or asymmetrical synapses on VIP-LIR dendrites or dendritic spines (Fig. 6d). Immunoreactive processes surrounded by astroglial processes were infrequently detected (Fig. 6e).

\section{VP-LIR neuronal elements}

VP-LIR neurons located mainly in the dorsomedial portion of the SCN contained well developed rER, polysomes and mitochondria. Their nuclei were oval shaped and contained dispersed chromatin. Immunoreactive dense granules of about 100-120 nm in diameter were also found in the cytoplasm (Fig. 7a). The dense population of astroglial processes which were observed around the VIP-LIR perikarya were not found around the VP-LIR neuronal perikarya. Therefore VP-LIR neuronal perikarya were usually surrounded by neuropiles. VP-LIR axons containing synaptic vesicles, mitochondria and immunoreactive dense granules of the same size as seen in the neuronal perikarya, were occasionally observed to make axo-dendritic synapses on non-immunoreactive dendrites (Fig. 7b). However, VP-LIR neuronal processes were seen far less than those with VIPLIR immunoreactivity. We could hardly detect VP-LIR axons to make synaptic contact with either non-immunoreactive or immunoreactive neuronal perikarya. We also occasionally found immunoreactive processes containing many immunoreactive dense granules intermingled with myelinated axons within the optic chiasm (Fig. 7c). On the other hand, non-immunoreactive axons, containing synaptic vesicles and mitochondria were frequently seen to make axo-somatic or axo-dendritic synapses on VP-LIR neuronal perikarya or dendrites particularly in the dorsomedial SCN (Fig. 8a, b).

\section{Discussion}

The distribution of VIP-LIR and VP-LIR neuronal elements in the monkey SCN has been delineated by light and electron microscopic immunocytochemistry. The dimension of the SCN of Japanese monkey (Macaca fuscata) in the present study is quite similar to that of other primate species such as squirrel monkey and rhesus monkey examined by computer graphic reconstruction using the sections stained with cresyl violet [15]. 

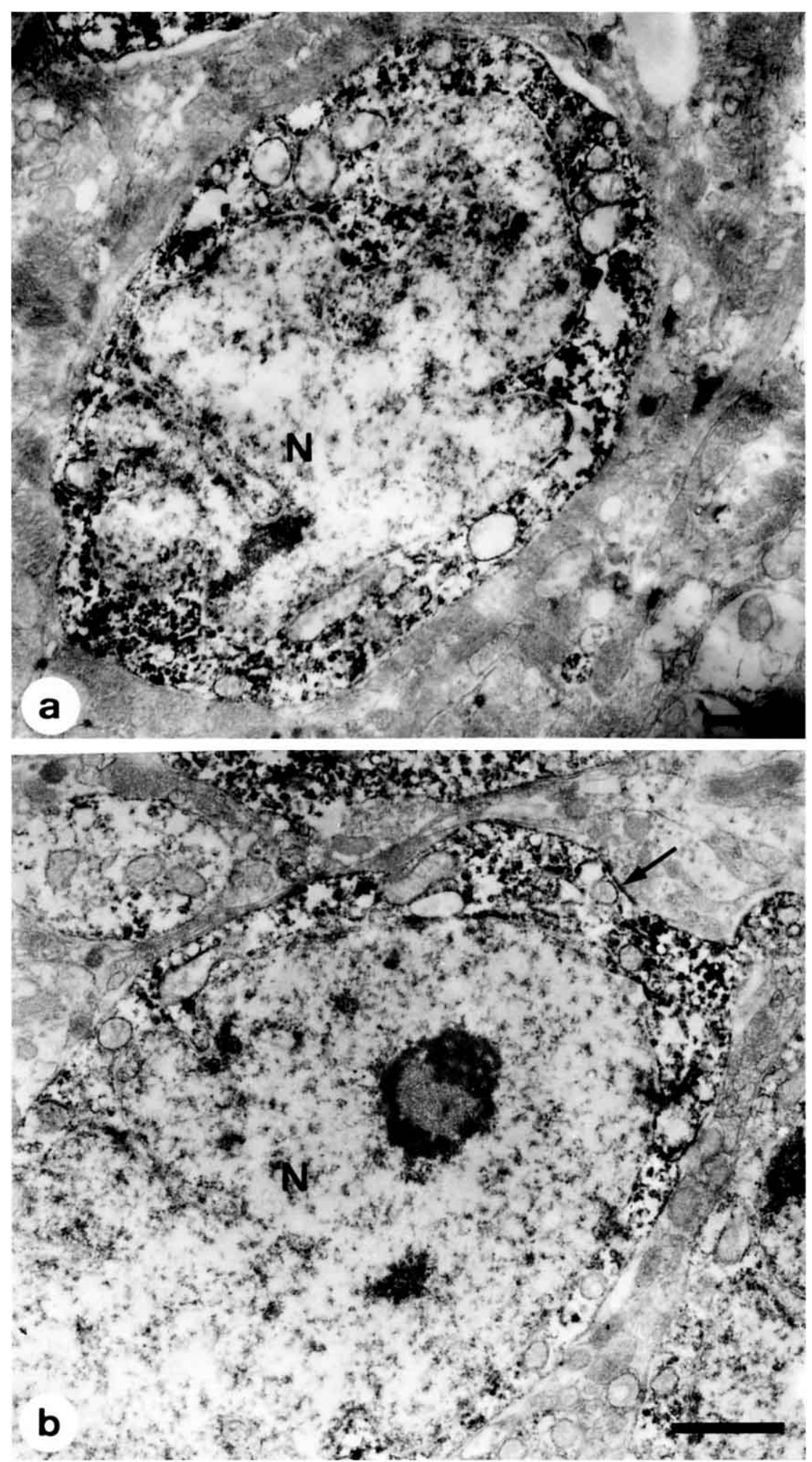

Fig. 4. a: VIP-LIR neuronal perikaryon with well developed cell organelles such as rER and mitochondria as well as immunoreactive dense granules is surrounded by astroglial processes. b: Non-immunoreactive axon containing synaptic vesicles and mitochondria makes an axosomatic synapse (arrow) on the surface of VIP-LIR neuronal perikaryon. $\mathrm{N}$, nucleus. Bar=1 $\mu \mathrm{m}$. 

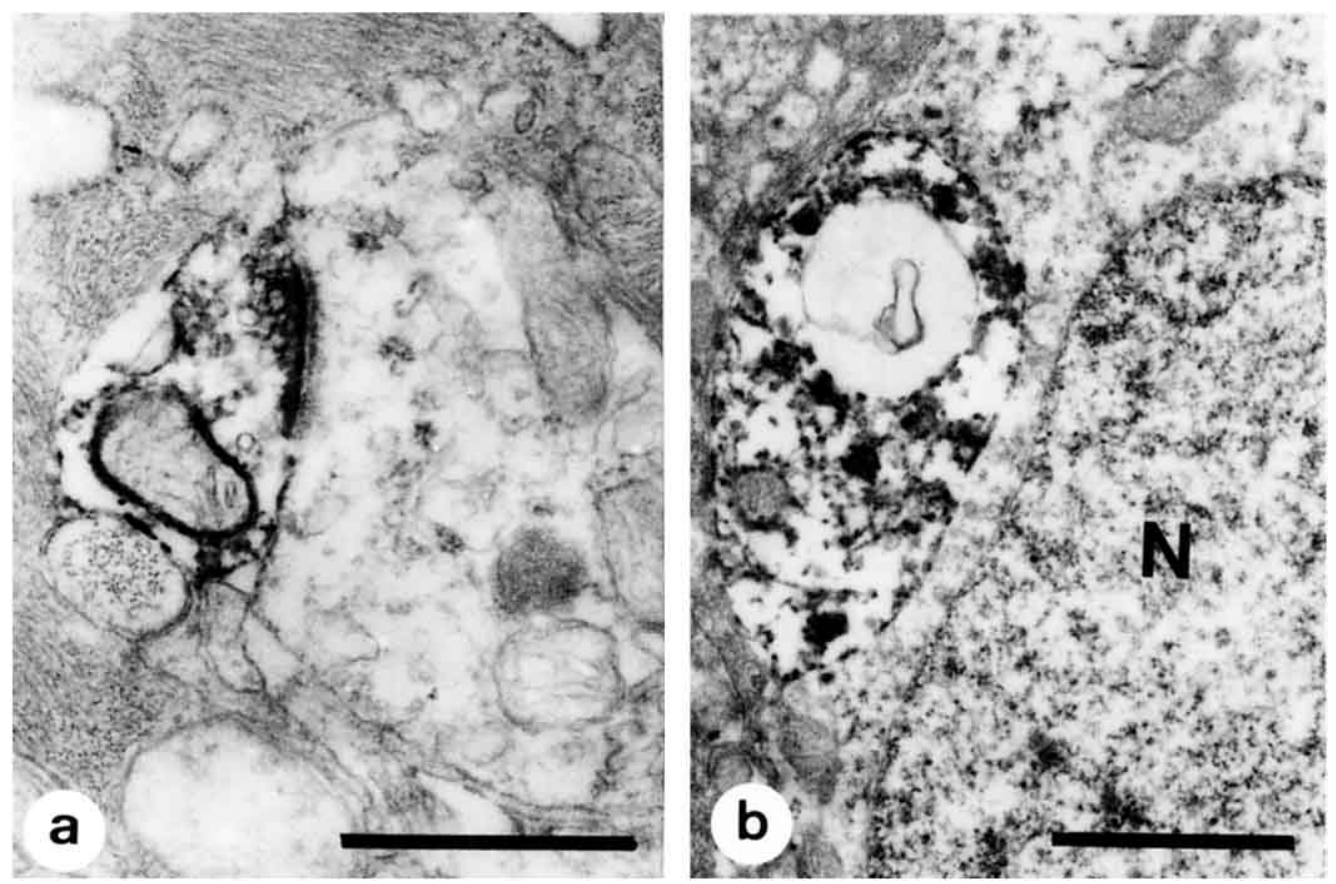

Fig. 5. a: VIP-LIR axon containing synaptic vesicles and mitochondria makes an asymmetrical axo-dendritic synapse on non-immunoreactive dendrite. b: VIP-LIR axon containing synaptic vesicles, mitochondria as well as immunoreactive dense granules in contact with non-immunoreactive neuronal perikaryon. $\mathrm{N}$, nucleus. $\mathrm{Bar}=1 \mu \mathrm{m}$.

\section{Distribution of VIP-LIR and VP-LIR neuronal elements}

The pattern of distribution of VIP-LIR and VP-LIR neuronal perikarya of the monkey $\mathrm{SCN}$ is fundamentally similar to that of the rat; VIP-LIR neurons are seen in the ventrolateral portion and VP-LIR neurons in the dorsomedial portion. However, there are subtle differences. VIPLIR neuronal perikarya in the monkey SCN are located more laterally in the mediolateral component in the central portion of this nucleus compared with the rat. The extent of the monkey SCN has a greater lateral expansion than that of the rat. On the other hand, there is a thinner distributional pattern in the dorsoventral direction, just above the optic chiasm, than in the rat. We also found VIP-LIR neuronal perikarya intermingled with myelinated axons in the optic chiasm. Their processes extend to the SCN among the myelinated axons of the optic chiasm in a fashion previously observed in the rat optic chiasm [3].

With respect to the VP-LIR neuronal perikarya, these elements in the monkey SCN show a more broad distribution particularly in the dorsomedial direction than those in the rat SCN. Their immunoreactivity is far less than that of the rat [7].

In the human SCN, the distribution of VIP-LIR and VP-LIR neuronal elements have been demonstrated by immunocytochemistry [16, 24]. Compared with VIP-LIR neuronal perikarya reported by Stopa et al. [24], their distribution in the monkey SCN of the present study is extremely similar. However, it is considerably different from that of the study of the human SCN by Mai et al. [16], in which fairly large number of VIP-LIR neurons were located in an area distant from the optic chiasm as well as in the region just above the optic chiasm. VP-LIR neuronal perikarya in the monkey SCN, on the other hand, show a similar distributional pattern to that of human SCN in both reports $[16,24]$ being located in the dorsomedial portion.

In addition to the ordinary distribution of VIP-LIR neuronal perikarya in the monkey SCN, a few small bipolar VIP-LIR neurons (the size of which is similar to those in the ventromedial portion) were detected along third ventricle in the periventricular nucleus continuous with the SCN. A few small VP-LIR neurons were also scattered in the same region. However, their immunoreactivity was either similar to that of magnocellular VP-LIR neurons or weaker than that of VP-LIR neurons in the SCN. The location of those small immunoreactive VP-LIR neurons is continuous with the periventricular nuclei in which magnocellular VP-LIR neuronal perikarya are distributed as had been shown by Kawata and Sano [12]. These VIP-LIR and VP-LIR neurons were, for the first time, detected in the monkey SCN. The finding of such neurons has not been published for other mammalian species. Although their functional roles are obscure, these VIP-LIR neurons may play a role different from those neurons which are located in the ventrolateral portion and may be involved in the entrainment of circadian rhythms. The VP-LIR neurons demonstrating strong immunoreactivity may be different from the ordinary weakly staining VP-LIR neurons as seen in the SCN, and may be an extension of the magnocellular VP-LIR neurons from the periventricular and paraventricular nuclei. However, the weakly immunoreactive neurons are considered to belong 

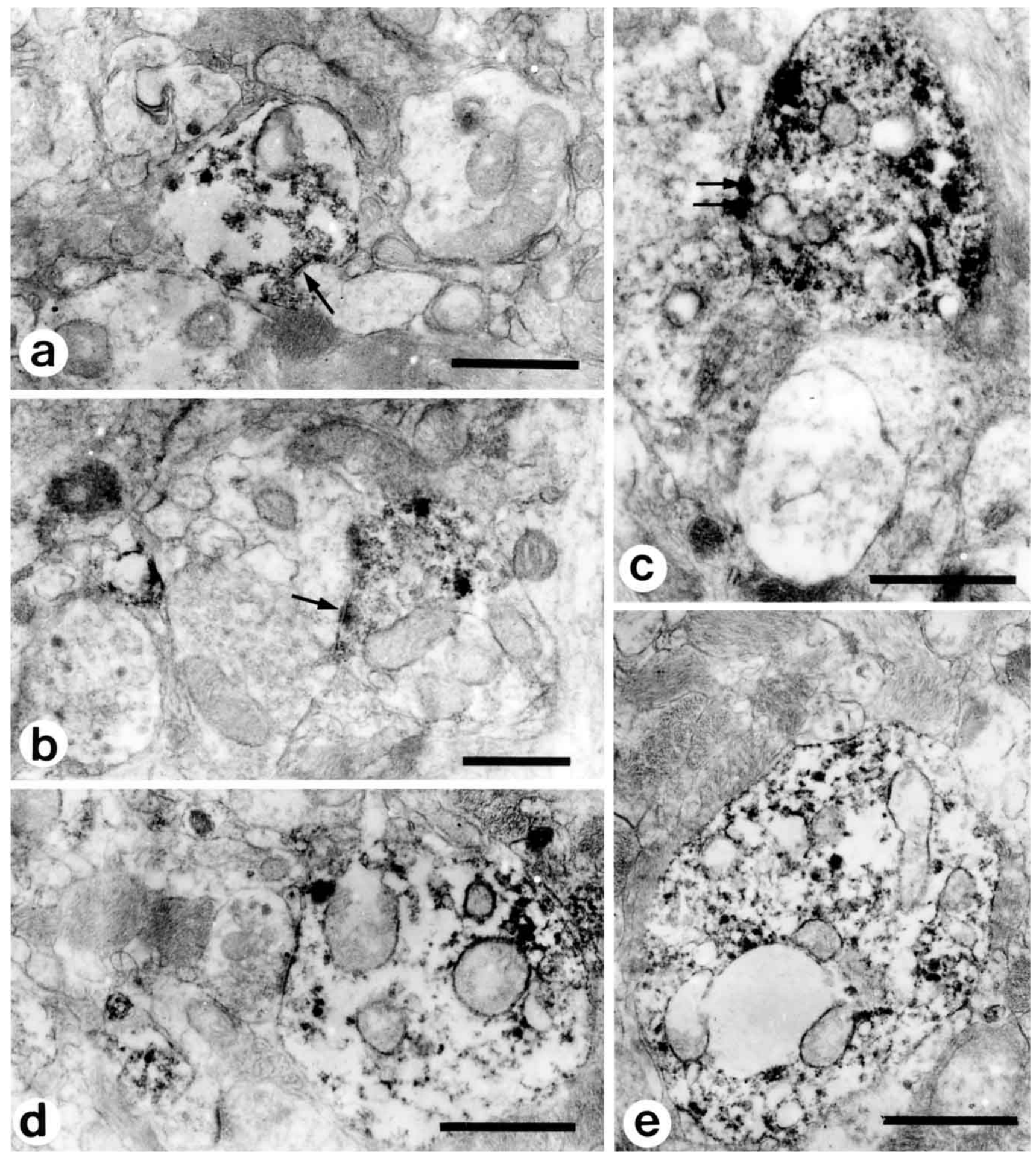

Fig. 6. a: VIP-LIR axon containing synaptic vesicles, mitochondria as well as dense granule makes an axo-spinodendritic synapse on nonimmunoreactive dendritic spine (arrow). b: VIP-LIR axon containing synaptic vesicles and mitochondria as well as dense granules terminates as an axo-axonic synapse on non-immunoreactive axon containing synaptic vesicles and mitochondria. Synaptic specialization is clearly visible between them (arrow). c: Synaptic contact between two VIP-LIR neuronal elements. The presynaptic axon seems to be a process with stronger immunoreactivity. d: Small non-immunoreactive axon containing synaptic vesicles and mitochondria makes axo-dendritic synapse on VIP-LIR dendrite. e: Astroglial processes with glial filaments girdles VIP-LIR process except for some synaptic portions. Bar=1 $\mu \mathrm{m}$. 

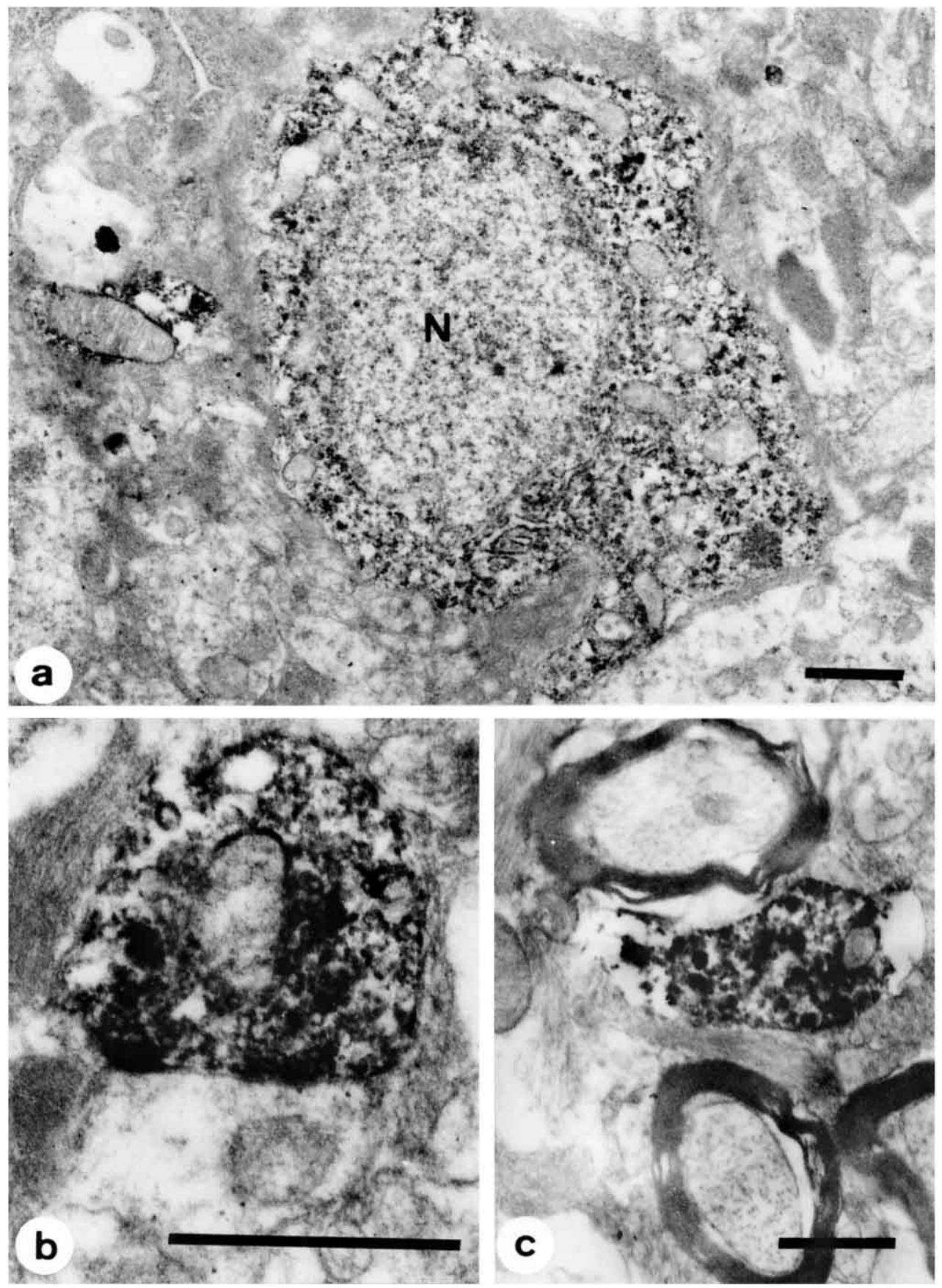

Fig. 7. a: VP-LIR neuronal perikaryon with well developed cell organelles such as rER and mitochondria as well as immunoreactive dense granules. N, nucleus. b: VP-LIR axon containing synaptic vesicles, mitochondria and dense granules makes an axo-dendritic synapse on nonimmunoreactive dendrite. c: VP-LIR axon containing immunoreactive dense granules is found among myelinated fibers in the optic chiasm. Bar $=1 \mu \mathrm{m}$.

to the VP-LIR neuronal population of the SCN. Numerous VIP-LIR processes and terminals seen as brown strands and dots were scattered throughout the SCN and were particularly noticeable in the dorsal areas. VP-LIR neuronal processes were mainly distributed in the dorsomedial portion of the SCN and were intermingled with the VP-LIR neuronal perikarya.

It has been clarified that VIP-like immunoreactivity and mRNA of VIP-LIR substance show diurnal variation under the influence of light from the retina $[1,5,21,25]$ and mRNA of VP-LIR substance show circadian rhythm without the influence of light in the rat SCN [28]. Therefore it is prerequisite to scrutinize whether or not similar phenomena occur in the VIP-LIR and VP-LIR neurons in the monkey SCN.

Recently the mouse circadian clock gene has been 

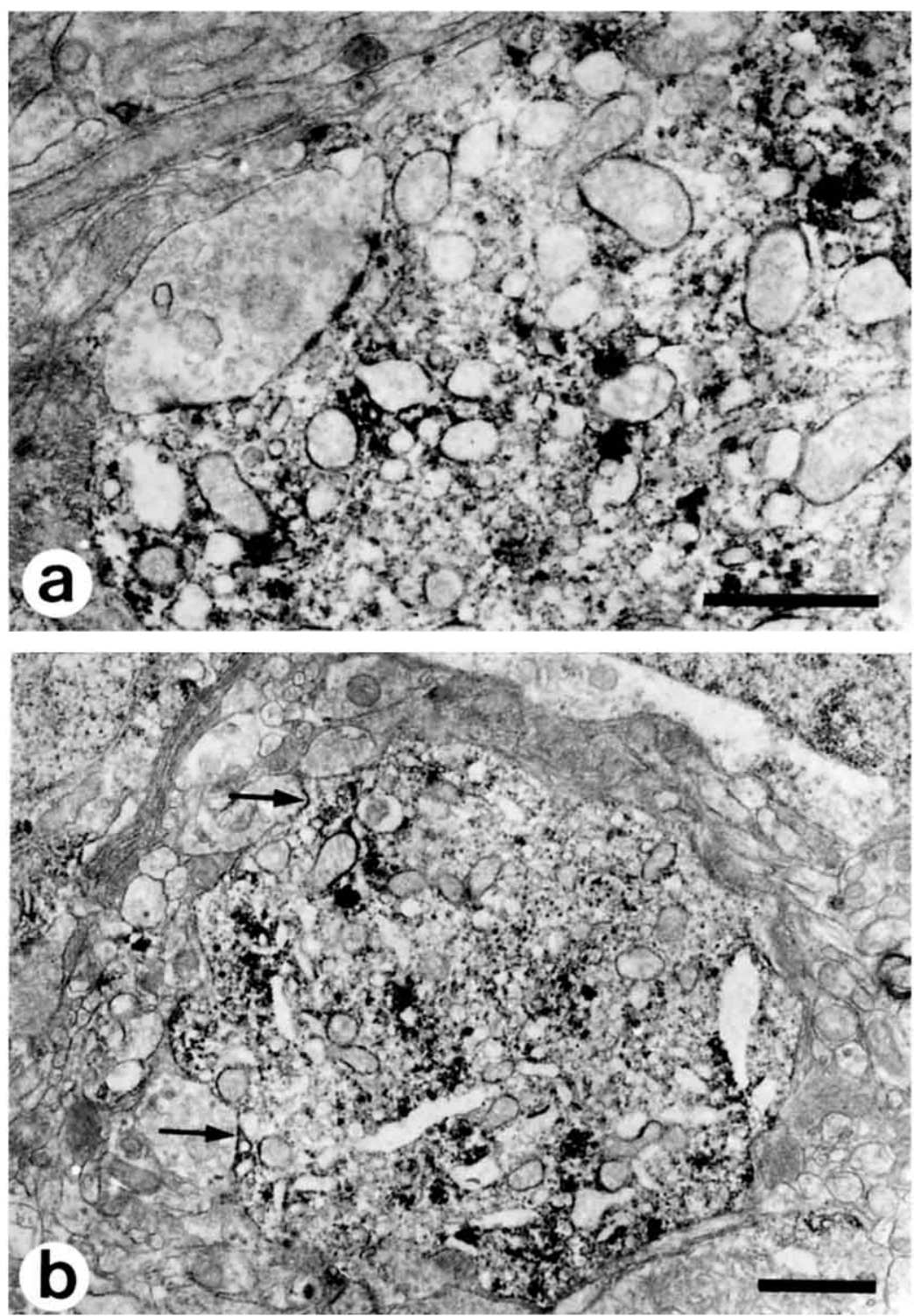

Fig. 8. a: Non-immunoreactive axon containing synaptic vesicles and mitochondria makes an axo-somatic synapse on the surface of VP-LIR neuronal perikaryon. b: Some non-immunoreactive axons containing synaptic vesicles and mitochondria make synapses on the surface of VP-LIR proximal dendrite (arrow). Bar=1 $\mu \mathrm{m}$.

cloned and mammalian homologue of period gene has also been cloned in the mouse and human brain and termed mPer and hPer [27]. Moreover, several characteristics of the mouse circadian clock gene and mPer has been clarified in the SCN $[2,13,22]$. Therefore, such kinds of genes and their functional characteristics in the primate SCN should be investigated in near future.

\section{Fine structure of VIP-LIR and VP-LIR neuronal elements}

VIP-LIR neuronal perikarya had well developed cell organelles such as mitochondria, rER and polysomes. Immunoreactive dense granules were also scattered in the cytoplasm. We also observed VIP-LIR neurons with well development of cell organelles and dense granules among the myelinated fibers of the optic chiasm. Those neurons showed a stronger immunoreactivity than seen in the VIPLIR neuronal perikarya located above the optic chiasm.

The most characteristic and interesting finding is that few axo-somatic synapses were found on the surface of VIPLIR neuronal perikarya, whereas the most of the VIP-LIR neuronal perikarya were surrounded by astroglial processes. On the other hand, non-immunoreactive axons were easily detected making axo-dendritic synapses on VIP-LIR dendrites. Therefore VIP-LIR neurons are believed to receive synaptic inputs mainly from other neuronal elements via axo-dendritic synapses.

We found VIP-LIR axons making both symmetrical or asymmetrical synapses on non-immunoreactive dendrites, the number of the latter is larger than the former. Asymmetrical synapses on non-immunoreactive dendritic spines 
were also found particularly in the dorsal portion of the SCN where numerous VIP-LIR processes were located. Similar findings have been reported in the rat SCN by Card et al. [3]. From the above findings, the VIP-LIR neurons are considered to be at least partially intrinsic neurons as well as those having projections to other CNS area.

On the other hand, we rarely observed VIP-LIR axons terminating on non-immunoreactive neuronal perikarya as definite axo-somatic synapses. Although some VIP-LIR processes still formed intimate contacts, but not clear synapses with non-immunoreactive neuronal perikarya.

VP-LIR neuronal perikarya showed well developed cell organelles such as mitochondria, rER and dense granules. We encountered VP-LIR neuronal processes containing dense granules, some of which made axo-dendritic synapses on non-immunoreactive dendrites. It is suggested, therefore, that VP-LIR neurons may be also partially intrinsic neurons in the SCN. Non-immunoreactive axons making axo-somatic or axo-dendritic synapses on VP-LIR neuronal perikarya or dendrites were particularly located in the dorsomedial portion of the SCN. It is suggested, therefore, that VP-LIR neurons receive inputs on both their perikarya and dendrites from other neuronal elements.

In the rat SCN, VIP-LIR neuronal elements have been shown to receive direct neuronal inputs from the retina by immunoelectron microscopy after eye enucleation [6]. The axons of the retinal ganglion cells have been shown to make synapses on the both VIP-LIR neuronal soma and dendrites using by double labeling immunoelectron microscopy [26]. Moreover it has been proven that VIP-LIR axons make synaptic contacts with VP-LIR neuronal perikarya and dendrites $[4,8]$. Therefore it is important to examine the fine structural interaction between the retinal ganglion cells and VIP-LIR neuronal elements and between the VIP-LIR and VP-LIR neuronal elements in the monkey SCN. Efferent neuronal projections of the monkey $\mathrm{SCN}$ should also be examined to determine if they are like those in the rat $\mathrm{SCN}$ as has been demonstrated by Watts and Swanson [30].

\section{Acknowledgment}

This work was supported in part by a grant from the Ministry of Education, Science, Sports and Culture, Japan.

\section{References}

1. Albers, H. E., Stopa, E. G., Zoeller, R. T., Kauer, J. S., King, J. C., Fink, J. S., Mobtaker, H. and Wolfe, H. (1990) Day-night variation in prepro vasoactive intestinal peptide/peptide histidine isoleucine mRNA within the rat suprachiasmatic nucleus. Brain Res. Mol. Brain Res. 7; 85-89.

2. Antoch, M. P., Song, E. J., Chang, A. M., Vitaterna, M. H., Zhao, Y., Wilsbacher, L. D., Sangoram, A. M., King, D. P., Pinto, L. H. and Takahashi, J. S. (1997) Functional identification of the mouse circadian Clock gene by transgenic BAC rescue. Cell 89; 655667.

3. Card, J. P., Brecha, N., Karten, H. J. and Moore, R. Y. (1981) Immunocytochemical localization of vasoactive intestinal polypeptide-containing cells and processes in the suprachiasmatic nucleus of the rat: light and electron microscopic analysis. $J$. Neurosci. 1; 1289-1303.

4. Daikoku, S., Hisano, S. and Kagotani, Y. (1992) Neuronal associations in the rat suprachiasmatic nucleus demonstrated by immunoelectron microscopy. J. Comp. Neurol. 325; 559-571.

5. Gozes, I., Shani, Y., Liu, B. and Burbach, J. P. H. (1989) Diurnal variation in vasoactive intestinal peptide messenger RNA in the suprachiasmatic nucleus of the rat. Neurosci Res. Commum. 5; 83-86.

6. Ibata, Y., Takahashi, Y., Okamura, H., Kawakami, F., Terubayashi, H., Kubo, T. and Yanaihara, N. (1989) Vasoactive intestinal peptide (VIP)-like immunoreactive neurons located in the rat suprachiasmatic nucleus receive a direct retinal projection. Neurosci. Lett. 97; 1-5.

7. Ibata, Y., Ichitani, Y., Tanaka, M., Hojo, T., Okamura, H. and Yanaihara, N. (1992) Light and electron microscopic immunocytochemistry of VIP neurons in the suprachiasmatic nucleus of the Japanese monkey. Biomed. Res. 13; 59-62.

8. Ibata, Y., Tanaka, M., Ichitani, Y., Takahashi, Y. and Okamura, H. (1993) Neuronal interaction between VIP and vasopressin neurones in the rat suprachiasmatic nucleus. Neuroreport $4 ; 128$ 130 .

9. Ibata, Y., Tanaka, M., Tamada, Y., Hayashi, S., Kawakami, F., Takamatsu, T., Hisa, Y. and Okamura, H. (1997) The suprachiasmatic nucleus: A circadian oscillator. The Neuroscientist 3; 215225.

10. Ibuka, N. and Kawamura, H. (1975) Loss of circadian rhythm in sleep-wakefulness cycle in the rat by suprachiasmatic nucleus lesions. Brain Res. 96; 76-81.

11. Johnson, R. F., Morin, L. P. and Moore, R. Y. (1988) Retinohypothalamic projections in the hamster and rat demonstrated using cholera toxin. Brain Res. 462; 301-312.

12. Kawata, M. and Sano, Y. (1982) Immunohistochemical identification of the oxytocin and vasopressin neurons in the hypothalamus of the monkey (Macaca fuscata). Anat. Embryol. (Berl) 165; $151-167$.

13. King, D. P., Zhao, Y., Sangoram, A. M., Wilsbacher, L. D., Tanaka, M., Antoch, M. P., Steeves, T. D., Vitaterna, M. H., Kornhauser, J. M., Lowrey, P. L., Turek, F. W. and Takahashi, J. S. (1997) Positional cloning of the mouse circadian clock gene. Cell 89; 641-653.

14. Kiss, J., Leranth, C. and Halasz, B. (1984) Serotoninergic endings on VIP-neurons in the suprachiasmatic nucleus and on ACTHneurons in the arcuate nucleus of the rat hypothalamus. A combination of high resolution autoradiography and electron microscopic immunocytochemistry. Neurosci. Lett. 44; 119-124.

15. Lydic, R., Albers, H. E., Tepper, B. and Moore-Ede, M. C. (1982) Three-dimensional structure of the mammalian suprachiasmatic nuclei: a comparative study of five species. J. Comp. Neurol. 204; 225-237.

16. Mai, J. K., Kedziora, O., Teckhaus, L. and Sofroniew, M. V. (1991) Evidence for subdivisions in the human suprachiasmatic nucleus. J. Comp. Neurol. 305; 508-525.

17. Moore, R. Y. and Eichler, V. B. (1972) Loss of a circadian adrenal corticosterone rhythm following suprachiasmatic lesions in the rat. Brain Res. 42; 201-206.

18. Moore, R. Y. and Lenn, N. J. (1972) A retinohypothalamic projection in the rat. J. Comp. Neurol. 146; 1-14.

19. Moore, R. Y., Halaris, A. E. and Jones, B. E. (1978) Serotonin neurons of the midbrain raphe: ascending projections. J. Comp. Neurol. 180; 417-438.

20. Moore, R. Y. (1983) Organization and function of a central nervous system circadian oscillator: the suprachiasmatic hypothalamic nucleus. Fed. Proc. 42; 2783-2789.

21. Okamoto, S., Okamura, H., Miyake, M., Takahashi, Y., Takagi, S., Akagi, Y., Fukui, K., Okamoto, H. and Ibata, Y. (1991) A diurnal variation of vasoactive intestinal peptide (VIP) mRNA 
under a daily light-dark cycle in the rat suprachiasmatic nucleus. Histochemistry 95; 525-528.

22. Shigeyoshi, Y., Taguchi, K., Yamamoto, S., Takekida, S., Yan, L., Tei, H., Moriya, T., Shibata, S., Loros, J. J., Dunlap, J. C. and Okamura, H. (1997) Light-induced resetting of a mammalian circadian clock is associated with rapid induction of the mPer1 transcript. Cell 91; 1043-1053.

23. Stephan, F. K. and Zucker, I. (1972) Circadian rhythms in drinking behavior and locomotor activity of rats are eliminated by hypothalamic lesions. Proc. Natl. Acad. Sci. U S A 69; 1583-1586.

24. Stopa, E. G., King, J. C., Lydic, R. and Schoene, W. C. (1984) Human brain contains vasopressin and vasoactive intestinal polypeptide neuronal subpopulations in the suprachiasmatic region. Brain Res. 297; 159-163.

25. Takahashi, Y., Okamura, H., Yanaihara, N., Hamada, S., Fujita, S. and Ibata, Y. (1989) Vasoactive intestinal peptide immunoreactive neurons in the rat suprachiasmatic nucleus demonstrate diurnal variation. Brain Res. 497; 374-377.
26. Tanaka, M., Ichitani, Y., Okamura, H., Tanaka, Y. and Ibata, Y. (1993) The direct retinal projection to VIP neuronal elements in the rat SCN. Brain Res. Bull. 31; 637-640.

27. Tei, H., Okamura, H., Shigeyoshi, Y., Fukuhara, C., Ozawa, R., Hirose, M. and Sakaki, Y. (1997) Circadian oscillation of a mammalian homologue of the Drosophila period gene. Nature $389 ; 512-516$.

28. Uhl, G. R. and Reppert, S. M. (1986) Suprachiasmatic nucleus vasopressin messenger RNA: circadian variation in normal and Brattleboro rats. Science 232; 390-393.

29. van den Pol, A. N. and Tsujimoto, K. L. (1985) Neurotransmitters of the hypothalamic suprachiasmatic nucleus: immunocytochemical analysis of 25 neuronal antigens. Neuroscience 15; 10491086.

30. Watts, A. G. and Swanson, L. W. (1987) Efferent projections of the suprachiasmatic nucleus: II. Studies using retrograde transport of fluorescent dyes and simultaneous peptide immunohistochemistry in the rat. J. Comp. Neurol. 258; 230-252. 